\title{
Conformal radiotherapy computation by the method of alternating projections onto convex sets
}

\author{
Shinhak Lee $\dagger$, Paul S Cho $\$$, Robert J Marks II $\dagger$ and Seho Oh\| \\ $\dagger$ Department of Electrical Engineering, University of Washington, Seattle, WA 98195-2500, \\ USA \\ $\ddagger$ Department of Radiation Oncology, University of Washington School of Medicine, \\ Box 356043, Seattle, WA 98195-6043, USA \\ || NeoPath Incorporated, 8271 154th Avenue NE Redmond, WA 98052, USA \\ Received 18 March 1996, in final form 10 February 1997
}

\begin{abstract}
Synthesis of beam profiles for a given dose prescription is a central problem in radiotherapy. Care must be taken in the beam design to expose the tumour volume at a high level, to avoid significant irradiation of critical organs, and to minimize exposure of all other tissue. Use of the synthesis procedure known as alternating projections onto convex sets (POCS) is shown to be a viable approach to beam design. POCS is a powerful tool for signal and image restoration and synthesis. Convex sets of signals obeying desired constraint sets are first specified. Then, by repeated projections onto these sets, convergence is to a signal obeying all desired constraints if the constraint sets have a finite intersection. In this paper we apply the method of POCS to conformal radiotherapy dose computation. The performance of the method is shown through three representative examples.
\end{abstract}

\section{Introduction}

An important objective of radiotherapy is to maximize the ratio of the dose delivered to the tumour to that which is deposited in the adjacent normal tissues. Conformal radiotherapy attempts to accomplish this by arranging the radiation sources in a configuration to best achieve the given dose distribution. In external beam therapy it is commonly envisioned that the source positions and intensities are varied dynamically by means of a computer controlled gantry and multileaf collimators. Successful implementation of conformal therapy depends, among other things, on treatment plan optimization.

All proposed solutions of the treatment plan optimization thus far require an iterative search. During each iteration a new estimate of beam intensity distribution is computed and its contribution evaluated. The process is repeated until the difference between the calculated and the desired dose distribution is minimized. The estimate of beam weights may be computed either stochastically (Webb 1989, Morrill et al 1990, Mageras and Mohan 1993, Rosen et al 1995) or deterministically (Brahme 1988, Bortfeld et al 1990, Holmes et al 1991). While the results appear to be similar between the two approaches, they differ dramatically in comparative computational complexity. The stochastic algorithms demand a number of operations that are typically several orders of magnitude greater than the deterministic models. For reasons of computational speed and clinical feasibility, computationally efficient algorithms are desirable.

$\S$ Author to whom correspondence should be addressed. 
In this paper we present a deterministic technique based on the method known as alternating projections onto convex sets (POCS). Following a general introduction to POCS we describe how to formulate the inverse radiotherapy problem in terms of convex sets and associated projection operators. Finally, the algorithm is applied to a number of representative cases in conformal therapy.

\section{POCS overview}

Alternating projections onto convex sets (POCS) $\dagger$ (Stark 1987, Youla and Webb 1982) is a powerful technique of signal recovery and synthesis. POCS was apparently first reported by Bregman (1965) and Gubin et al (1967) and was popularized by Youla and Webb (1982) and Stark (1987).

A set, $C$, is convex if $\boldsymbol{x}_{1} \in C$ and $\boldsymbol{x}_{2} \in C$ implies that $\lambda \boldsymbol{x}_{1}+(1-\lambda) \boldsymbol{x}_{2} \in C$ for all $0 \leqslant \lambda \leqslant 1$. Geometrically, as illustrated in figure $1(a)$, this means that the line segment connecting $x_{1}$ and $x_{2}$ is totally subsumed in the set $C$. The set shown in figure $1(b)$ is not convex because there are two points within the set that, when connected, result in a line segment that lies partially outside of the set. Examples of geometrical convex sets include balls, boxes, lines, line segments, cones and planes.

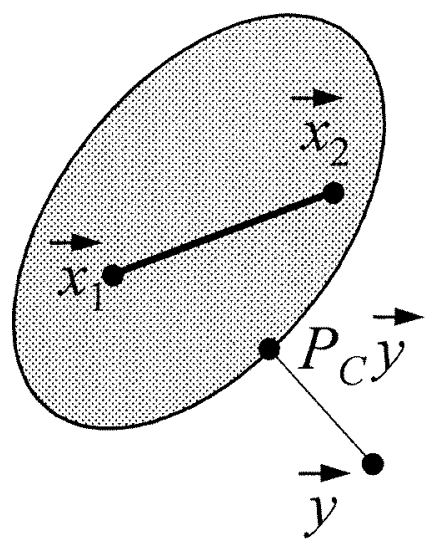

(a)

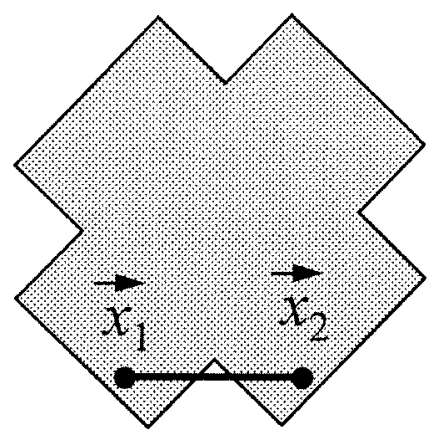

(b)

Figure 1. Illustration of the definition of a convex set and convex set projections. In (a), a set $C$ consists of all of the points in and on the oval. No matter what two elements of $C$ are chosen (here $x_{1}$ and $x_{2}$ ), the line segment connecting them lies totally within the set $C$. The set in $(a)$ is convex. The set of points in $(b)$ is not convex since, as shown, there are elements $\boldsymbol{x}_{1}$ and $\boldsymbol{x}_{2}$ in the set that produce a line segment that lies partially outside the set. The projection onto a convex set is shown in $(a)$. For an arbitrary point, $y$, the projection onto the convex set, $C$, (denoted $P_{C} \boldsymbol{y}$ ) is the (unique) closest point to $\boldsymbol{y}$ in the mean square sense. If $\boldsymbol{y}$ is already in the set, then the projection operation gives $y$, i.e. the point does not move.

For a given $y \notin C$, the projection onto $C$ is the unique vector in $C$ such that the mean square distance between $\boldsymbol{y}$ and its projection is minimum $\ddagger$. The projection of $\boldsymbol{y}$ onto set $C$

$\dagger$ The term, alternating traditionally does not appear in the POCS acronym.

$\ddagger$ The space is assumed throughout to be either $L_{2}$ or $\ell_{2}$. The mean square distance is the corresponding norm. For a more thorough discussion of POCS see Youla and Webb (1982), Youla's chapter in Stark (1987), or the tutorial by Marks (1997). 
is denoted by $P_{C} \boldsymbol{y}$. If a set is convex, the projection is unique. If a point to be projected is in the set, the projection results in the same point (i.e. if $\boldsymbol{y} \in C$, then $P_{C} \boldsymbol{y}=\boldsymbol{y}$ ).

POCS assumes that the convex constraint sets intersect. If the intersection contains many points, the fixed point will be determined by factors such as initialization and the order of projections. The existance of a number of solutions is simply a reflection on design flexibility imposed by the dose prescription. If the sets do not intersect, the prescription dose cannot be satisfied exactly. Constraint sets that are 'close' may satisfy the constraints to a good approximation. Even if the sets do not intersect, the result from POCS can be useful (Youla and Velasco 1986, Marks 1997).

Note that POCS can be used to establish whether a number of convex sets have a common intersection. In many cases, including beam profile synthesis for prescribed dose distribution, establishing whether or not the sets intersect prior to application of POCS is difficult. If the sets do not intersect, the POCS iteration will eventually break into a limit cycle. The closeness of one set to another can be determined by calculating the mean square distance between projections when the limit cycle is reached. If the sets have a common intersection, POCS will converge to a point contained in the intersection of the sets as illustrated in figure 2.

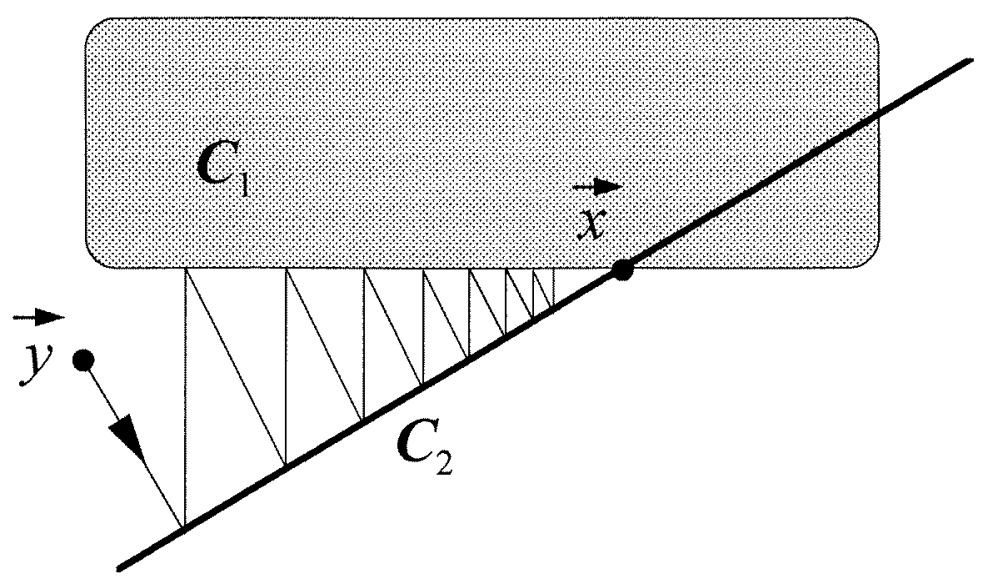

Figure 2. Alternately projecting between two or more convex sets with a non-empty intersection results in a limit point common to all sets (shown shaded here). Shown here are two convex sets of points. The set $C_{1}$ is the set of points in and on the rounded corner rectangle. Convex set $C_{2}$ is a line segment. Alternately projecting between the two sets results in convergence to the fixed point, $\boldsymbol{x}$, that lies in the intersection of the two convex sets. This fixed point can be different for a different starting point, $\boldsymbol{y}$, but will always lie on the intersection of the convex sets.

\section{Description of the beam vector synthesis algorithm}

The POCS method for synthesizing pencil beam weights to produce desired dose prescriptions consists of the following steps.

(i) Specify convex constraints the prescribed dose must satisfy. These constraints can originate from either dose prescription, physics or mathematical concerns.

(ii) Establish the projection onto each of these convex sets. 
(iii) Alternately project among the constraint sets. Iterate until convergence is achieved. The result is a beam profile synthesis for the dose prescription.

Design will be described and examples given for a two-dimensional cross section. Extension to the three dimensions is straightforward. Dose synthesis, an inherently continuous problem, is typically discretized to allow application of discrete analysis. Let $b(x, \theta)$, the beam intensity function, correspond to the intensity discretized at angle $\theta$ crossing the axis perpendicular to $\theta$ at the beam element position $x$. Assume there are $Q$ linear beam arrays at fixed equal angular intervals, $b_{k}(x)=b\left(x ; \theta=\frac{2 \pi}{Q} k\right)$ for $1 \leqslant k \leqslant Q$. The $k$ th array is discretized into $N$ pencil beam elements that can be expressed by the vector

$$
\boldsymbol{b}_{k}=\left[b_{k 1} b_{k 2} \cdots b_{k N}\right]^{T} .
$$

The same spatial interval between adjacent pencil beam elements is assumed. The dose domain is discretized into $M$ pixels. The dose contribution to the point $m$ from the $n$th beam element in the $k$ th beam array $\left(b_{k n}\right)$ is $\left(a_{m n}\right)_{k}$. The corresponding dose computation matrix, $\mathbf{A}_{k} \in R^{M \times N}$, is defined for $M$ tissue points. The dose from the $k$ th beam array is

$$
\begin{aligned}
\boldsymbol{d}_{k} & =\left[d_{k 1} d_{k 2} \ldots d_{k M}\right]^{T} \\
& =\mathbf{A}_{k} \boldsymbol{b}_{k}
\end{aligned}
$$

where $m$ th row and $n$th column element of $\mathbf{A}_{k}$ is $\left(a_{m n}\right)_{k}$. The contributions from all $Q$ beam arrays must be summed to give the total dose delivered to each pixel. Physically, the matrix $\mathbf{A}_{k}$ is the discretized kernel, $\mathbf{A}\left(\gamma, x, \frac{2 \pi}{Q} k\right)$ that specifies the dose to point $\gamma$ from the pencil beams in the $k$ th linear array. The vector, $\gamma$, is discretized into the $M$ pixels. This geometry is illustrated in figure 3 .

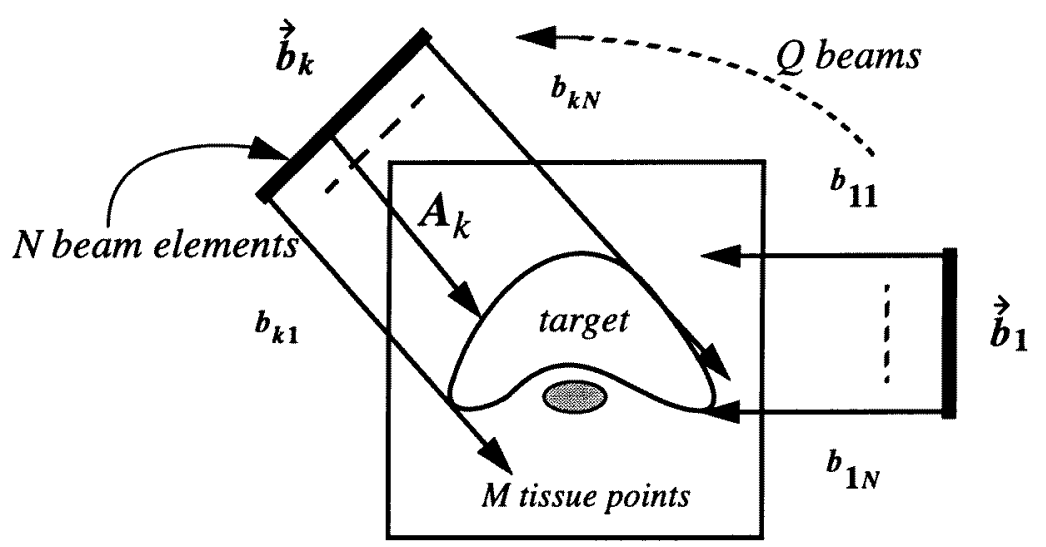

Figure 3. Geometry of the dose computation plane. There are $Q$ beam positions, each beam containing $N$ beam elements. The dose computation matrix corresponding to the $k$ th beam is A $_{k}$ whose dimension is $M \times N$, where $M$ is the number of tissue points.

For $Q$ discretized beam arrays, there are $Q$ dose vectors, $\left\{\boldsymbol{d}_{k} \mid 1 \leqslant k \leqslant Q\right\}$. These $Q$ vectors are stacked to form the parent dose vector

$$
\boldsymbol{d}=\left[\boldsymbol{d}_{1}^{T} \boldsymbol{d}_{2}^{T} \cdots \boldsymbol{d}_{Q}^{T}\right]^{T}
$$

where $d \in R^{M N \times 1}$. This is the space in which the dose constraint sets, all convex, are defined. The convexity of constraints and their corresponding projections are more easily established in this space than in the total dose space defined in equation (4) below. 
The total dose vector at $M$ tissue points, $t$, is the sum of dose vectors from every incident beam and can be computed from the parent dose vector in equation (3).

$$
\begin{aligned}
\boldsymbol{t} & =\left[t_{1} t_{2} \ldots t_{M}\right]^{T} \\
& =\sum_{k=1}^{Q} \boldsymbol{d}_{k} \\
& =\sum_{k=1}^{Q} \mathbf{A}_{k} \boldsymbol{b}_{k} .
\end{aligned}
$$

To synthesize the beam elements, the following constraint sets are used. Each is convex. The projection operation is given for each set. Proofs of convexity and projection for each case are given in the Appendix.

(i) Beam dose constraint set. Given the dose computation matrix, $\mathbf{A}_{k}$, and the beam vector, $\boldsymbol{b}_{k}$, for the $k$ th beam vector, the resulting dose vector is given by $\boldsymbol{d}_{k}=\mathbf{A}_{k} \boldsymbol{b}_{k}$. Generally, the dimension of the dose vector exceeds the number of beam elements. In other words, the dose vector has a larger number of degrees of freedom than the beam vector. The matrix, $\mathbf{A}_{k}$, is thus not full rank. Therefore, the beam vector cannot be obtained from direct inversion of the matrix when the beam vector is unknown. One standard way to solve for $\boldsymbol{b}_{k}$ is using pseudo-inversion. The resulting dose vector lies on the column space spanned by column vectors of $\mathbf{A}_{k}$. Pseudo-inverse solutions thus obtained are linear combinations of column vectors of $\mathbf{A}_{k}$ and form a convex set. The corresponding operator which gives one of these solutions is a projection operator. Mathematically, the convex set can be expressed as

$$
C_{B}=\left\{\boldsymbol{d} \mid \boldsymbol{d}_{k}=\mathbf{A}_{k} \boldsymbol{b}_{k}, 1 \leqslant k \leqslant Q\right\} .
$$

This operator projects the $k$ th vector component, $\boldsymbol{d}_{k}$ onto the column space of $\mathbf{A}_{k}$. The projection operator is

$$
P_{B} \boldsymbol{d}=\left(\left[P_{B} \boldsymbol{d}\right]_{1}^{T}\left[P_{B} \boldsymbol{d}\right]_{2}^{T} \ldots\left[P_{B} \boldsymbol{d}\right]_{Q}^{T}\right)^{T}
$$

where the projection of the $k$ th component is

$$
\left[P_{B} \boldsymbol{d}\right]_{k}=\mathbf{T}_{k} \boldsymbol{d}_{k}
$$

The matrix that projects on the column space of $\mathbf{A}_{k}$ is

$$
\mathbf{T}_{k}=\mathbf{A}_{k}\left(\mathbf{A}_{k}^{T} \mathbf{A}_{k}\right)^{-1} \mathbf{A}_{k}^{T} \text {. }
$$

(ii) Target dose constraint set. This constraint set requires the delivered dose match the prescribed dose in the target volume. Let $T$ denote a subset of numbers from 1 to $M$ corresponding to indices of the target volume. Let the prescribed dose vector be

$$
\boldsymbol{p}= \begin{cases}p_{k} & k \in T \\ 0 & \text { otherwise. }\end{cases}
$$

These values are determined by sampling the continuous prescribed dose. In our simulations, each $p_{k}$ is set to one. The target dose constraint set is then defined by

$$
C_{T}=\left\{\boldsymbol{d} \mid \sum_{i=1}^{Q} d_{i j}=\left\{\begin{array}{ll}
p_{j} & j \in T \\
\text { don't care } & \text { otherwise }
\end{array}\right\} .\right.
$$

The projection onto $C_{T}$ is

$$
P_{T} \boldsymbol{d}=\left(\left[P_{T} \boldsymbol{d}\right]_{1}^{T}\left[P_{T} \boldsymbol{d}\right]_{2}^{T} \ldots\left[P_{T} \boldsymbol{d}\right]_{Q}^{T}\right)^{T}
$$


where

$$
\left[P_{T} \boldsymbol{d}\right]_{k}=\boldsymbol{d}_{k}+\frac{1}{Q} \mathbf{I}_{T}\left(\boldsymbol{p}-\sum_{i=1}^{Q} \boldsymbol{d}_{i}\right) .
$$

The diagonal matrix, $\mathbf{I}_{T}$, serves as a spatial discriminator and is given by

$$
\left(\mathbf{I}_{T}\right)_{j j}= \begin{cases}1 & j \in T \\ 0 & \text { otherwise. }\end{cases}
$$

Thus only the projection components intersecting the target dose are affected.

(iii) Organ dose constraint set. This constraint set controls the dose in organs at risk where the dose must be kept low. Let $S$ denote a subset of numbers from 1 to $M$ corresponding to the indices of the critical organ region. The organ dose constraint set is

$$
C_{O}=\left\{\boldsymbol{d} \mid 0 \leqslant \sum_{i=1}^{Q} \sum_{j \in S} d_{i j} \leqslant E_{1}, \boldsymbol{d} \in C_{B}\right\}
$$

where $E_{1}$ is the upper limit of allowable integrated dose in the critical region. To present the projection, define the vector $\boldsymbol{r}$ with elements

$$
r_{k}= \begin{cases}1 & k \in S \\ 0 & \text { otherwise. }\end{cases}
$$

The corresponding projection operator is

$$
P_{O} \boldsymbol{d}=\left(\left[P_{O} \boldsymbol{d}\right]_{1}^{T}\left[P_{O} d\right]_{2}^{T} \ldots\left[P_{O} d\right]_{Q}^{T}\right)^{T}
$$

where

$$
\left[P_{O} \boldsymbol{d}\right]_{k}= \begin{cases}\mathbf{T}_{k}\left(\boldsymbol{d}_{k}+\frac{E_{1}-\sum_{j=1}^{Q} \boldsymbol{r}^{T} \mathbf{T}_{j} \boldsymbol{d}_{j}}{\sum_{j=1}^{Q} \boldsymbol{r}^{T} \mathbf{T}_{j} \boldsymbol{r}} \boldsymbol{r}\right) & \sum_{j=1}^{Q} \boldsymbol{r}^{T} \mathbf{T}_{j} \boldsymbol{d}_{j}>E_{1} \\ \mathbf{T}_{k}\left(\boldsymbol{d}_{k}-\frac{\sum_{j=1}^{Q} \boldsymbol{r}^{T} \mathbf{T}_{j} \boldsymbol{d}_{j}}{\sum_{j=1}^{Q} \boldsymbol{r}^{T} \mathbf{T}_{j} \boldsymbol{r}} \boldsymbol{r}\right) & \sum_{j=1}^{Q} \boldsymbol{r}^{T} \mathbf{T}_{j} \boldsymbol{d}_{j}<0 \\ \mathbf{T}_{k} \boldsymbol{d}_{k} & \text { otherwise. }\end{cases}
$$

(iv) Non-negative beam constraint. Physics dictates that each beam element have a non-negative value. This set requires adherence to this property. The corresponding convex non-negative beam constraint sets, one for each beam, are $\dagger$

$$
C_{k}=\left\{\boldsymbol{d} \mid \boldsymbol{d}_{k}=\mathbf{A}_{k} \boldsymbol{b}_{k}, \boldsymbol{b}_{k} \geqslant \mathbf{0}\right\} ; 1 \leqslant k \leqslant Q .
$$

The set $C_{k}$ can be expressed as the intersection of a number of convex component sets.

$$
C_{k}=\bigcap_{n=1}^{N} C_{k n} .
$$

The component sets are

$$
C_{k n}=\left\{\boldsymbol{d} \mid \boldsymbol{d}_{k}=\mathbf{A}_{k} \boldsymbol{b}_{k}, b_{k n} \geqslant 0\right\} ; 1 \leqslant n \leqslant N
$$

where $b_{k n}$ is the $n$th element of $\boldsymbol{b}_{k}$. For a given $\boldsymbol{d}$, the sign of each $b_{k n}$ can be determined from

$$
\boldsymbol{b}_{k}=\left(\mathbf{A}_{k}^{T} \mathbf{A}_{k}\right)^{-1} \mathbf{A}_{k}^{T} \boldsymbol{d}_{k} .
$$

$\dagger$ The notation $\boldsymbol{b}_{k} \geqslant \mathbf{0}$ means each element of $\boldsymbol{b}_{k}$ is non-negative. 
The projection onto $C_{k n}$ is

$$
P_{k n} \boldsymbol{d}_{k}= \begin{cases}\mathbf{T}_{k n} \boldsymbol{d}_{k} & b_{k n}<0 \\ \boldsymbol{d}_{k} & \text { otherwise }\end{cases}
$$

where the projection matrix is

$$
\mathbf{T}_{k n}=\mathbf{A}_{k n}\left(\mathbf{A}_{k n}^{T} \mathbf{A}_{k n}\right)^{-1} \mathbf{A}_{k n}^{T} .
$$

and the matrix $\mathbf{A}_{k n} \in R^{M \times(N-1)}$ is formed by removing the $n$th column of $\mathbf{A}_{k}$. In lieu of projecting onto each $C_{k}$, projection is done sequentially onto each $C_{k n}$ set. These projection operators can be concatenated into the single operator

$$
\Theta_{+}=\prod_{k=1}^{Q} \prod_{n=1}^{N} P_{k n} .
$$

Although $\Theta_{+}$is composed of a string of projection operators, it, itself is not a projection operator. It does not, for example, directly project onto the set

$$
C_{+}=\bigcap_{k=1}^{Q} C_{k} .
$$

After a projection operator component of $\Theta_{+}$is performed, the constraint corresponding to the previous projection operator component may no longer be satisfied. Thus, after $\Theta_{+}$ is applied, some of the beam elements can still be negative. However, the magnitude of negative weights will diminish as iteration progresses and become negligible as it will be shown in section 5 .

Using these four convex constraint sets and corresponding projections, the dose can be synthesized using POCS. Let $l$ be the POCS iteration counter. Let $\boldsymbol{b}_{i}[l]$ be the $i$ th beam and $d_{i}[l]$ be the $i$ th vector in $d[l]$ at the $l$ th iteration. Then $d[l+1]$ is obtained by the recursion

$$
d[l+1]=\Theta_{+} P_{B} P_{T} P_{O} d[l] .
$$

POCS will also converge if some projections are used more than others (Stark 1987). Less computationally intensive projections may thus be evaluated more frequently. For example, we found faster convergence occurred using

$$
d[l+1]=\Theta_{+}\left(P_{B} P_{T}\right)^{L} P_{O} d[l] .
$$

where $\left(P_{B} P_{T}\right)^{L}$ indicates $L$ repeated projections between two sets, $C_{A}$ and $C_{D}$ in each of the entire projection. We used $L=8$.

The POCS iterative process is terminated when the difference between the prescribed dose and the obtained total dose is sufficiently small.

The corresponding beam vector, $\boldsymbol{b}_{i}[l+1]$, can be uniquely determined using minimum mean square error solution

$$
\boldsymbol{b}_{i}[l+1]=\left(\mathbf{A}_{i}^{T} \mathbf{A}_{i}\right)^{-1} \mathbf{A}_{i}^{T} \boldsymbol{d}[l+1], 1 \leqslant i \leqslant Q .
$$

If there are any residual negative beam weights due to constraint set non-intersection or early iteration truncation, they are set to zero. We found that if there were any negative beam weights, they were relatively small in magnitude. 

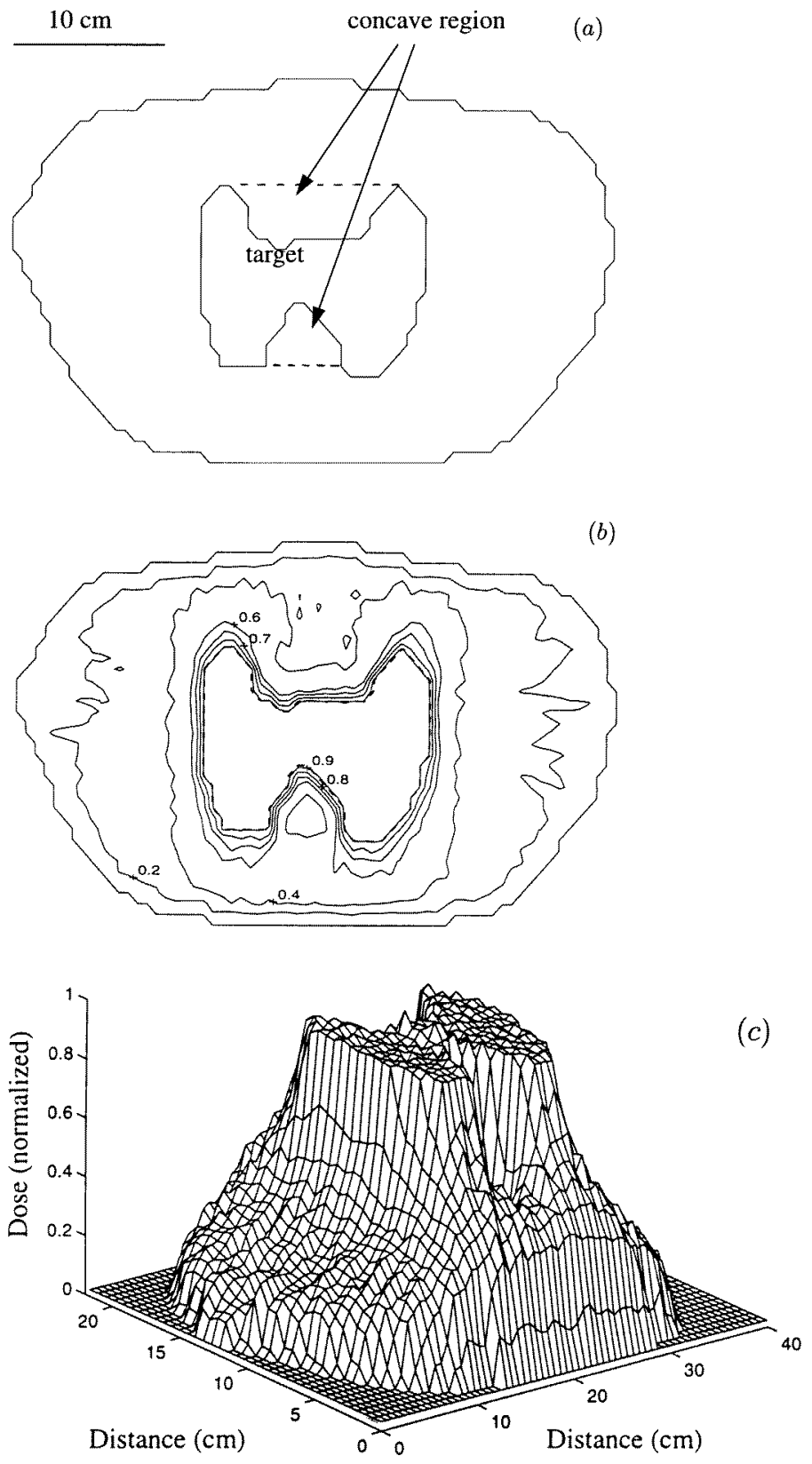

Figure 4. (a) Brahme's concave butterfly shaped tumour volume. The matrix size of the dose distribution plane is $35 \times 63$. (b) and (c) Results of POCS dose synthesis applied to Brahme's butterfly shaped tumour after 15 iterations. 31 beams, each containing 55 beam elements were used. (b) The isodose contours in full curves and the tumour contour in broken curves. (c) A 3D plot of the relative dose versus the transaxial coordinates.

\section{Application in conformal therapy}

POCS is now applied to three representative conformal radiotherapy optimization cases: 


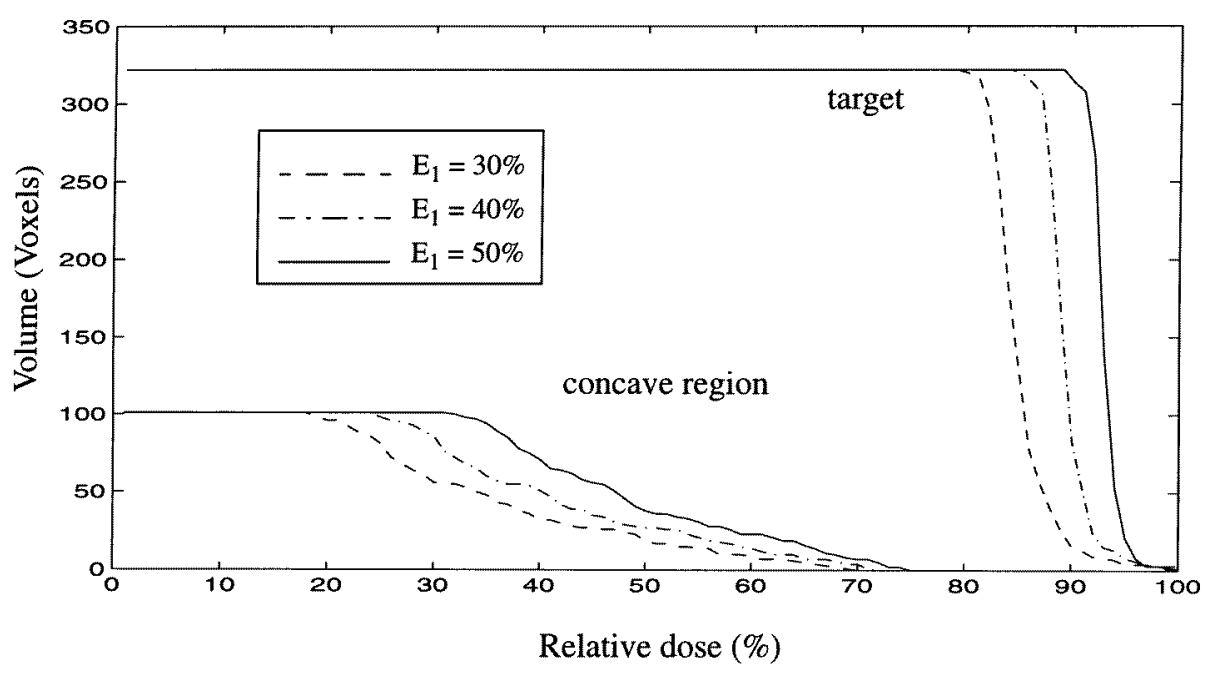

Figure 5. Dose-volume histograms illustrating the trade-off between tumour dose conformation and normal tissue sparing within the concave regions. The number of beams and the number of POCS iterations were kept constant at 31 and 15, respectively, while the dose constraint parameter was varied from $30 \%$ to $50 \%$.

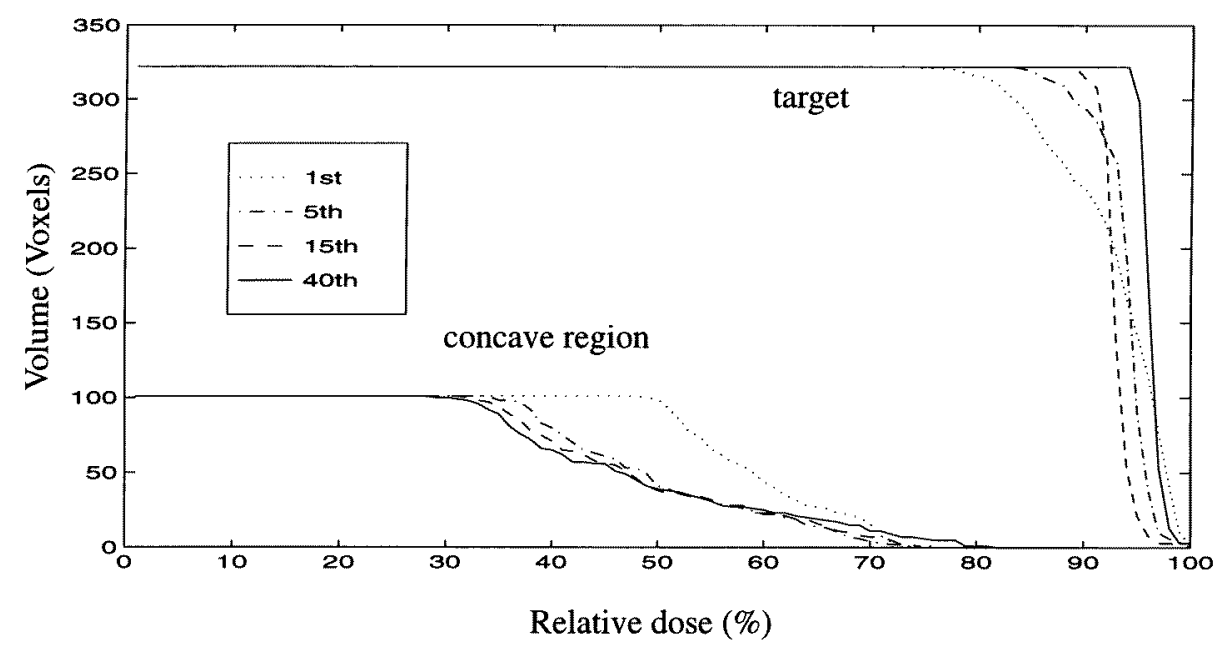

Figure 6. Same as figure 5 except, in this case, the dose constraint was fixed at $50 \%$ while the number of iteration was varied from 1 to 40 .

(i) A target with concave regions,

(ii) A target that surrounds a sensitive organ, and

(iii) A target adjacent to large volumes of organs at risk.

Dose at a sample point from a pencil beam element is calculated using the approximate formula

$$
D(l)=\operatorname{TPR}(l, w) \times \operatorname{ISC}(l)
$$

where $l$ is the depth of calculation. TPR is the tissue-phantom ratio at depth, $l$, for the 


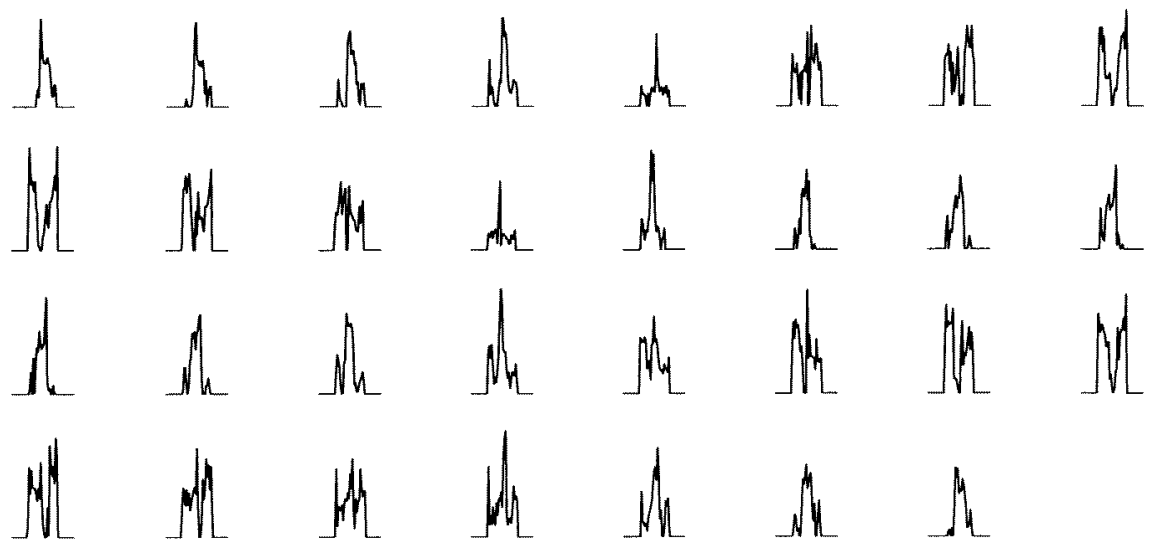

Figure 7. Beam intensity profiles synthesized for Brahme's geometry. The upper left profile corresponds to the beam which is incident horizontally from the right side of figure $4(a)$. The gantry rotates in a counterclockwise direction. Each beam consists of 55 elements spanning the width of the external contour.

equivalent square field size, $w$, and ISC is the inverse square correction of the primary fluence due to beam divergence. The external and internal organ contours are assumed to be constant in the axial direction. The height of the target volume is equated to the maximum target width. To increase the computational efficiency, the dose contributions from each pencil beam having a unit weight are pre-computed and stored in a file. The value of dose computation matrix elements is simply the value of $D(l)$ with corresponding depth, $l$. The x-ray photon energy of the beam is set at $18 \mathrm{MV}$. Beam synthesis is performed for a two-dimensional slice through the tumour volume. The POCS beam optimization technique can easily be extended to three dimensions at a cost of additional computation.

\subsection{Target with concave regions}

The first example is Brahme's butterfly shaped tumour volume (Brahme 1988). This target shape, illustrated in figure 4(a), presents a potential challenge for treatment planning. The difficulty arises because of the concave normal tissue regions above and below the tumour. Without intensity modulation, uniform delivery of the prescribed dose to the target can be achieved only at the expense of undesirable escalation of dose to the concave regions. The maximum width and height of the external contour are $40 \mathrm{~cm}$ and $22 \mathrm{~cm}$, respectively, and corresponds to 63 by 35 tissue elements. Thirty-one equiangularly spaced beams, each containing 55 elements, are used.

The results of inverse planning after 15 iterations using the POCS algorithm are shown in figures $4(b)$ and $4(c)$. Sharp dose gradients around the target border are observed. The organ dose constraint specified in terms of integrated limiting dose, $E_{1}$, to the concave regions was set to $50 \%$ of the maximum value. As shown here and in the third example, the organ dose constraint parameter can be used to control the trade-off between the tumour dose and the dose in concave region. For example, if the dose to the target needs to be maximized more uniformly, then a larger value should be set for the constraint parameter, as shown in figure 5 .

The dose-volume histogram, shown in figure 6, illustrates the effect of iteration number on dose distributions. As it can be seen, the dose level converges quickly to the specified 
$10 \mathrm{~cm}$

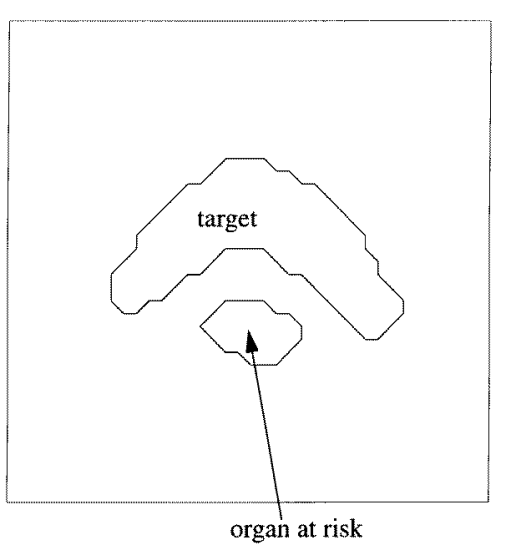

(a) (b)

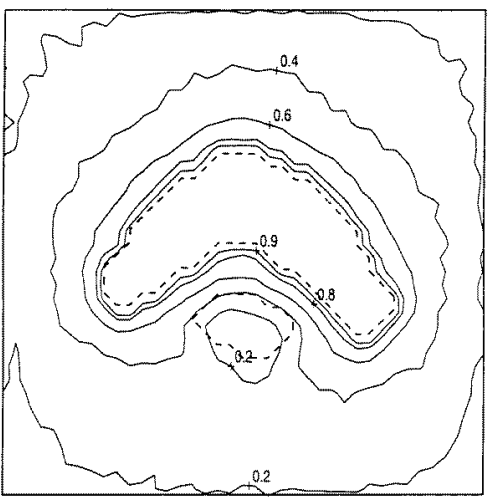

(c)

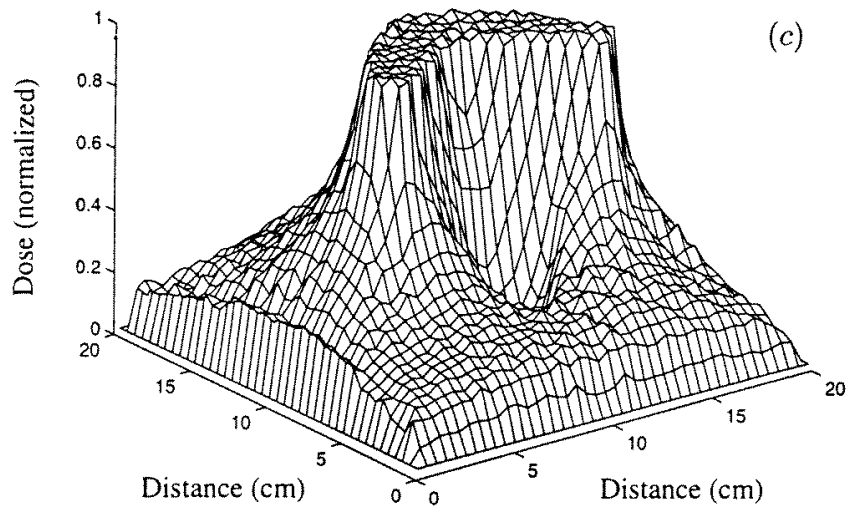

Figure 8. (a) Bortfeld's horseshoe-shaped target with an organ at risk. Matrix size of the dose distribution plane is $39 \times 39$. (b) and (c) Results of the POCS dose synthesis applied to Bortfeld's geometry after 15 iterations. Thirty one beams, each containing 55 beam elements were used. The value of $20 \%$ dose constraint was imposed on the critical structure. (b) The isodose contours in full curve and the tumour-organ contours in broken curve. (c) A 3D plot of the relative dose versus the transaxial coordinates.

level in the concave regions. For the target volume, the convergence is more gradual. No appreciable improvement is observed for number of iterations beyond 40 . The intensity profiles of the incident beams after 15 iterations are shown in figure 7.

\subsection{Target with a sensitive organ at risk}

The second example is adopted from Bortfeld et al (1990). As shown in figure 8(a), the irradiation volume consists of a horseshoe-shaped target and a dose limiting organ within the concave region. The computation results after 15 iterations using 31 beams with 55 elements each and a $20 \%$ organ dose constraint are shown in figures $8(b)$ and $8(c)$. The dose falls off sharply outside the target and dips further near the critical organ. The corresponding beam intensity modulation profiles are shown in figure 9 .

Next, we vary the number of beams in steps from 3 to 72 while limiting the integrated 


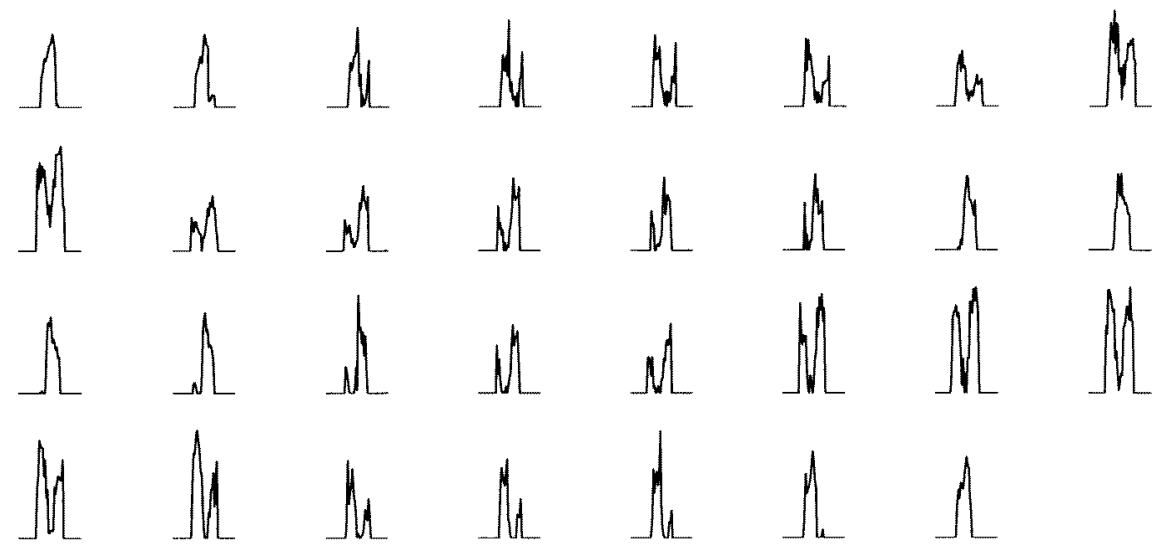

Figure 9. Beam intensity profiles obtained for Bortfeld's geometry. The upper left profile corresponds to the beam which is incident horizontally from the right side of figure $8(a)$. The gantry rotates in a counterclockwise direction. Each beam consists of 55 elements spanning the width of the square external contour.

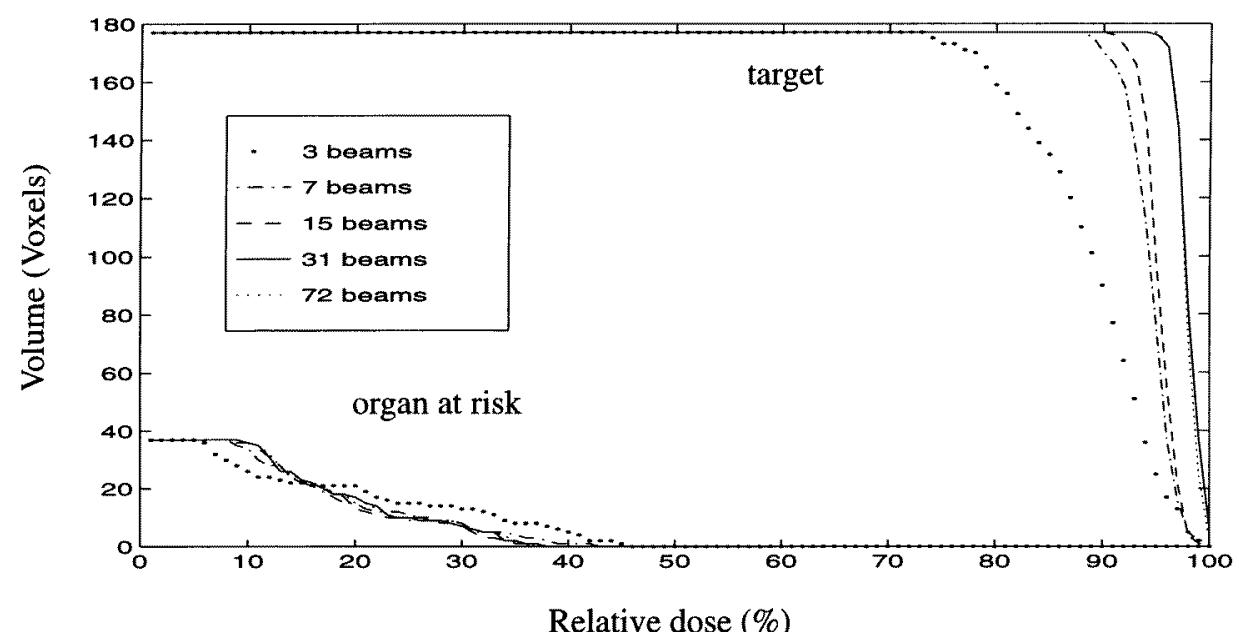

Figure 10. Dose-volume histograms showing the effect of increasing the number of beams from 3 to 72 in Bortfeld's problem. The number of iterations was kept constant at 15 .

organ dose to $20 \%$. The results after 15 iterations are plotted in figure 10 . As expected, the target dose conformation improves with an increase in the number of beams. The degree of improvement is, however, nonlinearly related to the number of added beams and eventually reaches a point of diminishing return.

\subsection{Target adjacent to large volumes of sensitive organs}

Our final example contains two organs that are large relative to the target, as shown in figure 11(a). The external contour width, height, dose matrix size, and the number of beam elements are the same as those used in section 4.1. The results after 30 iterations 

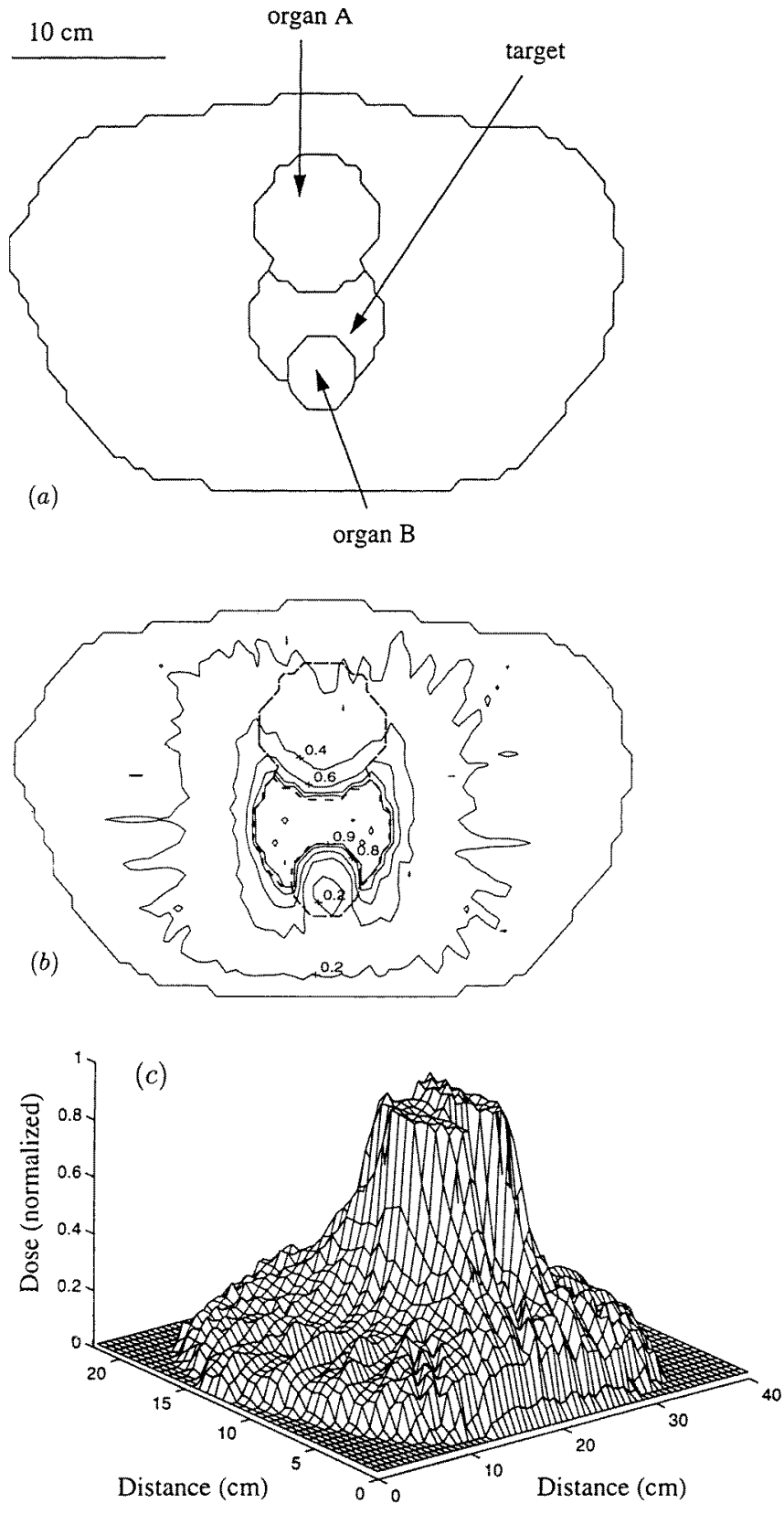

Figure 11. (a) Tumour that is adjacent to two relatively large organs at risk. The matrix size of the dose distribution plane is $35 \times 63$. (b) and (c) Results of the POCS dose synthesis applied to the geometry described in $(a) .31$ beams and 30 iterations were used. $(b)$ The isodose contours in full curve and the tumour-organ contours in broken curve. (c) A 3D plot of the relative dose versus the transaxial coordinates.

using 31 beams with a $30 \%$ organ dose constraint are shown in figures 11(b) and 11(c). The complex target-organ geometry demonstrates the difficulty in achieving acceptable treatment planning. Although we have applied a $30 \%$ value to both organ A and B, these values can 


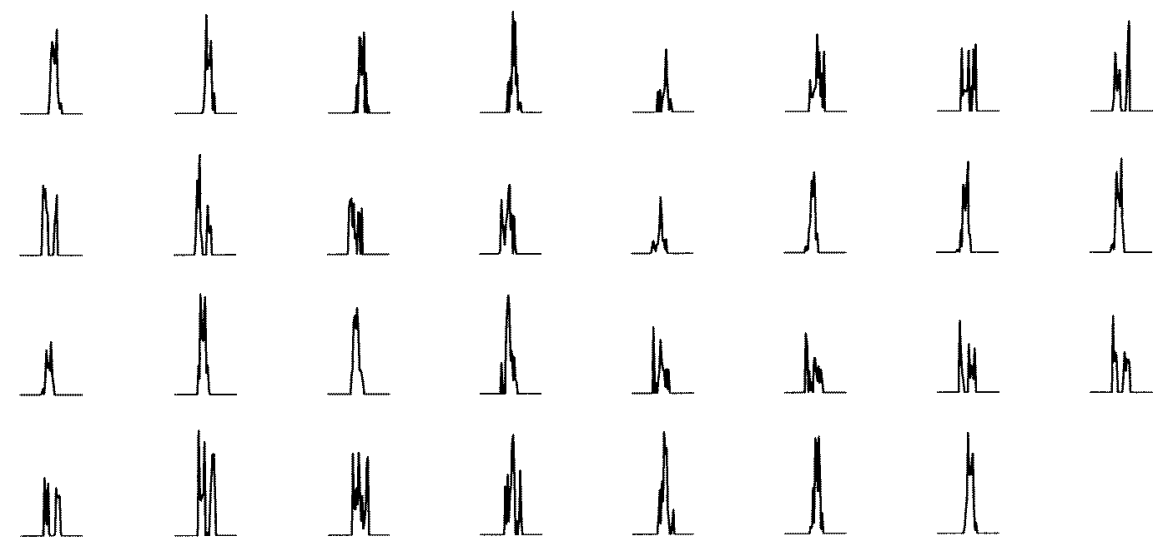

Figure 12. Beam intensity profiles obtained after 30 iterations for the geometry described in figure 11 $(a)$. The upper left profile corresponds to the beam which is incident horizontally from the right side of the figure 11 $(a)$. The gantry rotates in a counterclockwise direction. Each beam consists of 55 elements spanning the width of the external contour.

be varied independently according to the clinical requirement. The corresponding intensity profiles of the incident beams are shown in figure 12. In figure 13, the dose constraint of the critical organ was varied while keeping other parameters constant. The dose-volume histograms indicate that a low critical organ dose is achievable at the price of reduced dose conformation for the target.

\section{Discussion}

We have investigated the feasibility of applying the method of alternating projections onto convex sets (POCS) to solve the problem of inverse dose computation in conformal therapy. The results are very promising. The constraints commonly used in inverse treatment planning were successfully expressed as convex sets which, in turn, were used to synthesize the beam weights to a solution. The proposed method is applicable to manyvariable problems such as intensity modulation. Computation of large matrices is simplified through a decomposition technique. For instance, assume we are given a $1000 \times 1000$ dose computation matrix (A) and 10 beams. As illustrated in figure 14, the matrix decomposition yields submatrices $\left(\mathbf{A}_{1} \mathbf{A}_{2} \ldots \mathbf{A}_{10}\right)$. This allows for 10 inversions of a $100 \times 100$ matrix $\left(\left(\mathbf{A}_{k}^{T} \mathbf{A}_{k}\right)^{-1}, 1 \leqslant k \leqslant 10\right)$.

In this study the organ dose constraint was formulated to reduce the integrated dose to the organ. This is suitable for the parallel organ model. For the serial organ model, it is desirable to reduce the maximum dose rather than the overall dose. In this case, the convex constraint set is given by

$$
C_{O j}=\left\{d \mid 0 \leqslant \sum_{i=1}^{Q} d_{i j} \leqslant E_{2}, j \in S\right\}
$$

where $E_{2}$ is the maximum allowable organ dose. The corresponding projection for $i$ th pixel 


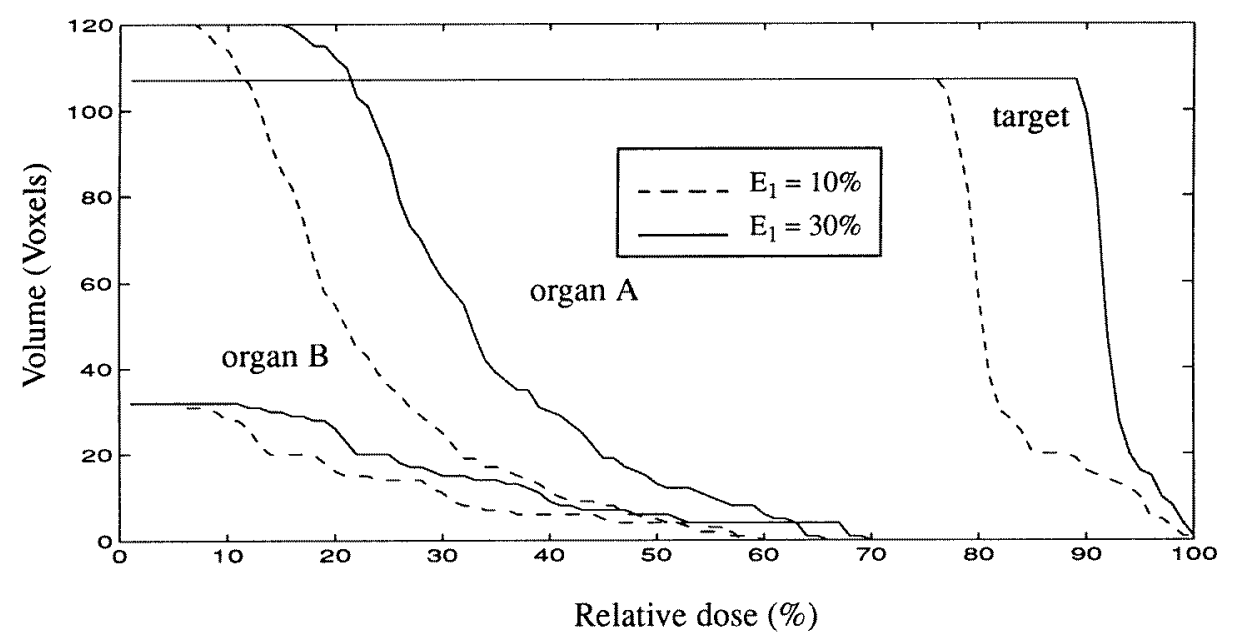

Figure 13. Dose-volume histograms demonstrating the effect of varying the critical organ dose constraint parameters. The number of beams and the number of iterations were held constant at 31 and 30 , respectively.

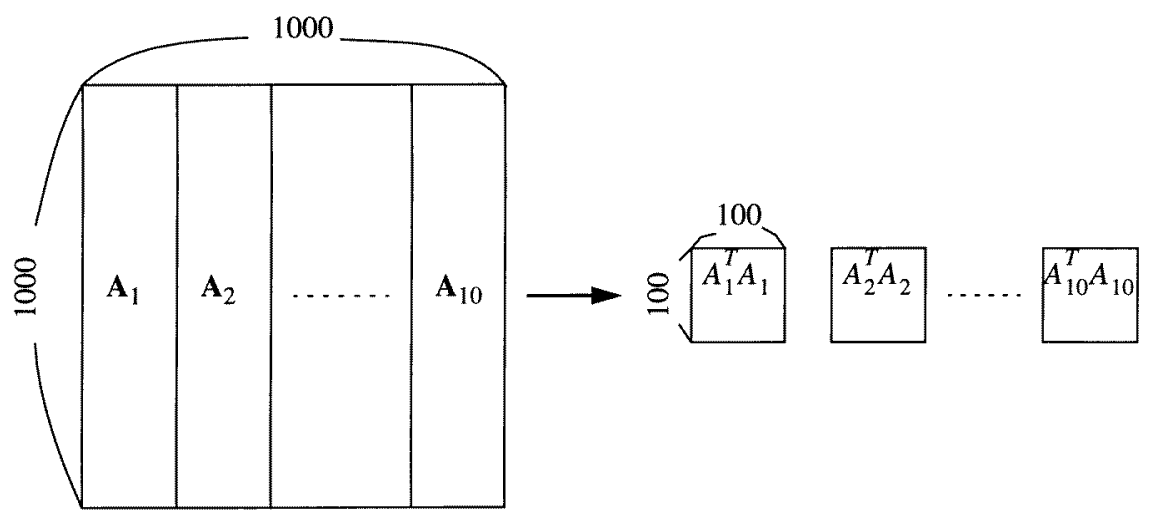

Figure 14. Illustration of how a large dose computation matrix $(\mathbf{A})$ is decomposed into submatrices $\left(\mathbf{A}_{1} \ldots \mathbf{A}_{10}\right)$ and inversion performed on small square matrices $\left(\mathbf{A}_{1}^{T} \mathbf{A}_{1}, \ldots, \mathbf{A}_{10}^{T} \mathbf{A}_{10}\right)$.

in the organ region is

$$
\left[P_{O i} d\right]_{k i}= \begin{cases}s_{i}^{T} \mathbf{T}_{k}\left(\boldsymbol{s}_{i}^{T} \boldsymbol{d}_{k}+\frac{E_{2}-\sum_{j=1}^{Q} s_{i}^{T} \mathbf{T}_{j} \boldsymbol{d}_{j}}{\sum_{j=1}^{Q} s_{i}^{T} \mathbf{T}_{j} s_{i}} \boldsymbol{s}_{i}\right) & \sum_{j=1}^{Q} \boldsymbol{s}_{i}^{T} \mathbf{T}_{j} \boldsymbol{d}_{j}>E_{2} \\ \boldsymbol{s}_{i}^{T} \mathbf{T}_{k}\left(\boldsymbol{d}_{k}-\frac{\sum_{j=1}^{Q} s_{i}^{T} \mathbf{T}_{j} \boldsymbol{d}_{j}}{\sum_{j=1}^{Q} s_{i}^{T} \mathbf{T}_{j} s_{i}} \boldsymbol{s}_{i}\right) & \sum_{j=1}^{Q} \boldsymbol{s}_{i}^{T} \mathbf{T}_{j} \boldsymbol{d}_{j}<0 \\ \boldsymbol{s}_{i}^{T} \mathbf{T}_{k} \boldsymbol{d}_{k} & \text { otherwise }\end{cases}
$$

where the elements of the vector, $s_{i} \in R^{M \times 1}$, are zero except at $i$ th pixel of the organ region. Depending on the type of organ, one can impose either of these constraint types, 
serial or parallel. The computation time will be longer for the serial organ model because the constraint set must be imposed for each sample point within the organ. For instance, the calculation time increased by $72 \%$ for the second example using 31 beams after 15 iterations.

The model of dose deposition considered only primary fluence. Incorporation of scattered radiation should not affect the ability of the POCS algorithm to provide an inverse solution, although the degree of conformation and beam modulation is likely to be different. It is reported that improved dose conformation of the target can be achieved when scatter is included in synthesis of beam intensity modulations (Chen et al 1995). It is expected that inclusion of scatter will increase the computation time per iteration. Without scattered radiation, dose to a sample point is interpolated from two nearest primary beam elements, thus requiring only $M \times 2 \times Q$ operations. On the other hand, if the scatter contributions are considered, the number of operations will increase to $M \times N \times Q$.

A question arises: to what point does POCS converge? The simple answer is: to a point satisfying all the constraints (assuming the constraint sets intersect). Quantitatively the point of convergence can be determined by calculating the mean square distance between projections. Application of the distance analysis on the three test cases reveals that although the constraint sets do not intersect they lie very close to one another. This is shown in figure 15. As the iteration progresses the distances between the sets decrease until convergence is achieved. The final convergence point relative to each set is illustrated in the rectangular box within the figure. It is apparent that the final convergence point depends on not only iteration number but also complexity of target-organ structure. All three cases exhibit similar convergence characteristics except for the distance to the non-negativity constraint set. Note that the second case satisfies the non-negativity constraint much better than the other two. This may be attributed to the presence of a transition area between the target and the organ at risk. In the other two cases, the target volumes are connected to the critical region demanding a sharp dose gradient at the structure boundary and therefore making it more difficult to satisfy the prescription. The distance to the non-negativity constraint set can be reduced at the expense of increasing the distance elsewhere.

All previously proposed beam optimization methods used objective function. In these methods, the nature of objective function determines the type of optimization technique that is suitable. If an objective function is known to have only a single minimum, a deterministic technique can be implemented. Otherwise, computationally expensive stochastic method such as simulated annealing capable of local minima detrapping must be used. In POCS, which does not use objective function, its applicability is determined by whether convex formalism of necessary constraints and associated projections exist. This alternative approach to the objective function minimization presents a new opportunity to explore the nature of the conformal therapy optimization problem. We are currently investigating the possibility of using POCS to solve optimization problems involving dosevolume constraints and biological response parameters, some of which are considered to have local minima solutions. Even in these cases, it may be possible to reformulate the problem in terms of convex expressions (Cho et al submitted).

\section{Conclusion}

We have demonstrated that the concept of alternating projections onto convex sets (POCS) can be successfully applied to the problem of intensity modulated conformal radiotherapy. The results indicate that the beam intensity profiles can be synthesized in 15 to 30 iterations, depending on the complexity of the tumour-organ geometry. Unlike other beam 

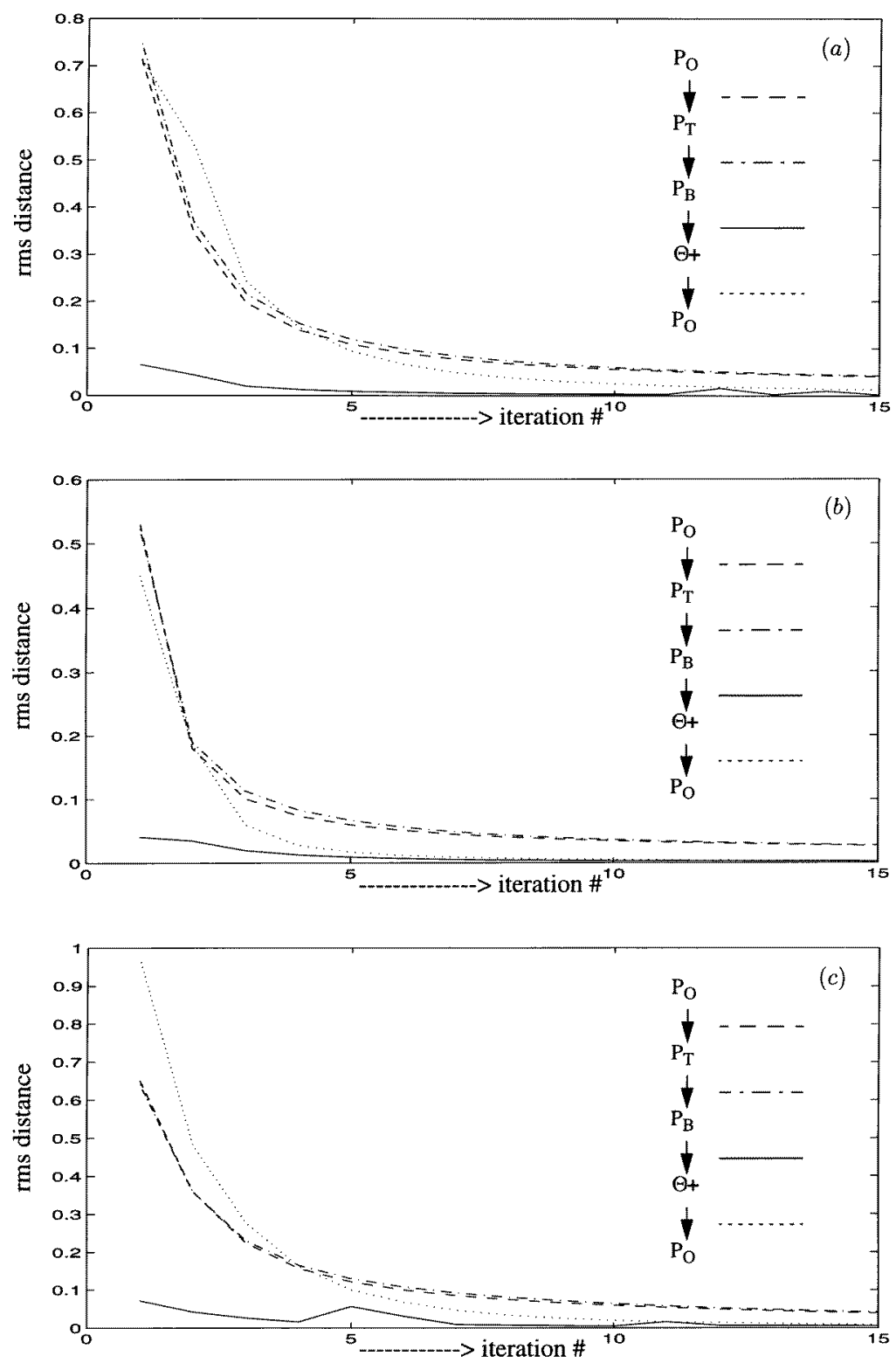

Figure 15. The point of POCS convergence can be determined by calculating the mean square distance between projections. The distance between two consecutive projections was calculated for each iteration. The distance alalysis for three test cases is shown in figures $(a),(b)$ and (c). (a) The total energy (the sum of squares) of the synthesized dose in this example is 18.76. POCS was truncated after 15 iterations on $C_{B}$, the column space of the dose computation matrix. The distance from this point to the set $C_{O}$ is 0.01 . The distance to set $C_{T}$ is 0.04 . The distance to $C_{+}$is 0.15 . (b) In this example, the total synthesized dose energy is 11.91 . The distances to $C_{O}, C_{T}$, and $C_{+}$are $0.002,0.026$ and 0.005 respectively. (c) The total energy here is 7.42 . The distances to $C_{O}, C_{T}$ and $C_{+}$are $0.002,0.02$ and 0.029 respectively.

optimization techniques, POCS does not rely on objective function minimization. As such, it provides a unique tool for conformal beam optimization research. 


\section{Appendix A. Proof of convexity and projection of $C_{B}$}

\section{Proof of convexity}

$C_{B}$ is a set of all vectors whose component vector, $\boldsymbol{d}_{i}$, can be represented as a linear combination of the columns of matrix, $\mathbf{A}_{i}$. Let $\boldsymbol{f}, \boldsymbol{g} \in C_{B}$ and $\boldsymbol{f}_{i}=\mathbf{A}_{i} \boldsymbol{b}_{i}, \boldsymbol{g}_{i}=\mathbf{A}_{i} \boldsymbol{h}_{i}$, respectively, for beam vectors, $\boldsymbol{b}_{i}$ and $\boldsymbol{h}_{i}$. We examine $\alpha \boldsymbol{f}_{i}+(1-\alpha) \boldsymbol{g}_{i}$.

$$
\begin{aligned}
\alpha \boldsymbol{f}_{i}+(1-\alpha) \boldsymbol{g}_{i} & =\alpha \mathbf{A}_{i} \boldsymbol{b}_{i}+(1-\alpha) \mathbf{A}_{i} \boldsymbol{h}_{i} \\
& =\mathbf{A}_{i}\left[\alpha \boldsymbol{b}_{i}+(1-\alpha) \boldsymbol{h}_{i}\right] .
\end{aligned}
$$

The resulting vector is simply another linear combination of columns of matrix, $\mathbf{A}_{i}$. Therefore, the set, $C_{B}$ is convex. Q.E.D.

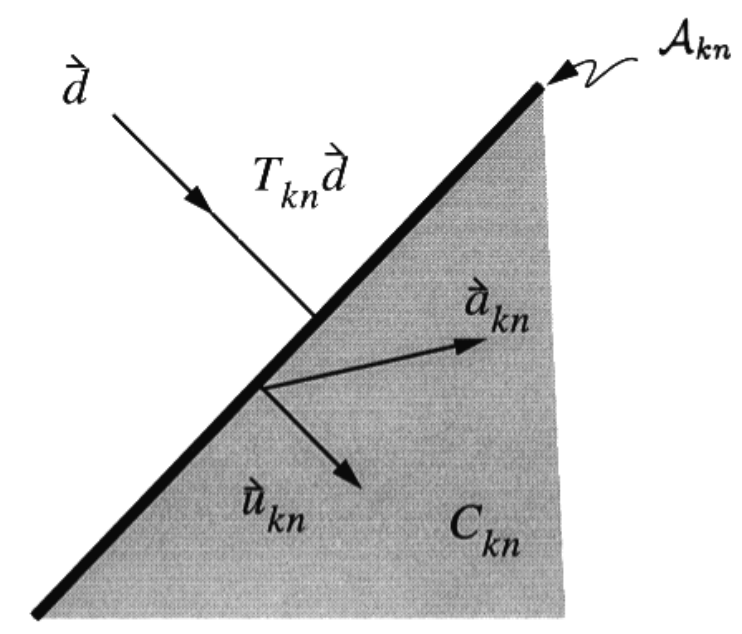

Figure A1. The subspace, $\mathcal{A}_{k n}$, divides the column space of $\mathbf{A}_{k}$, one for negative beam element $\left(b_{k n}\right)$ and the other (shaded area) for the positive beam element. If the dose vector $(\boldsymbol{d})$ does not lie in the positive half space, it is projected through the projection matrix, $\mathbf{T}_{k n}$.

\section{Proof of projection}

Let $J=J_{1}+J_{2}+\cdots+J_{Q}$ where

$$
J_{k}=\left\|\boldsymbol{d}_{k}-\mathbf{A}_{k} \boldsymbol{b}_{k}\right\|^{2} .
$$

$J$ is minimum when each $J_{k}$ is minimum. Differentiating $J_{k}$ w.r.t. $\boldsymbol{b}_{k}$ and setting it to zero gives minimum.

$$
\begin{aligned}
\frac{\partial J}{\partial \boldsymbol{b}_{k}} & =\mathbf{A}_{k}^{T}\left(\boldsymbol{d}_{k}-\mathbf{A}_{k}\right) \boldsymbol{b}_{k} \\
& =0 .
\end{aligned}
$$

Thus $\boldsymbol{b}_{k}=\left(\mathbf{A}_{k}^{T} \mathbf{A}_{k}\right)^{-1} \mathbf{A}_{k}^{T} \boldsymbol{d}_{k}$. Therefore, the projection of $\boldsymbol{d}$ is

$$
P_{B} \boldsymbol{d}=\left(\left[P_{B} \boldsymbol{d}\right]_{1}^{T}\left[P_{B} \boldsymbol{d}\right]_{2}^{T} \ldots\left[P_{B} \boldsymbol{d}\right]_{Q}^{T}\right)^{T}
$$

where $\left[P_{B} \boldsymbol{d}\right]_{k}=\mathbf{A}_{k}\left(\mathbf{A}_{k}^{T} \mathbf{A}_{k}\right)^{-1} \mathbf{A}_{k}^{T} \boldsymbol{d}_{k}$. Q.E.D. 


\section{Appendix B. Proof of convexity and projection of $C_{T}$}

Proof of convexity

Let $\boldsymbol{f}, \boldsymbol{g} \in C_{T}$. Next, we examine $s=\sum_{i=1}^{Q}\left(\alpha \boldsymbol{f}_{i}+(1-\alpha) \boldsymbol{g}_{i}\right)$.

$$
s=\alpha \sum_{i=1}^{Q} \boldsymbol{f}_{i}+(1-\alpha) \sum_{i=1}^{Q} \boldsymbol{g}_{i} .
$$

Since $\left[\sum_{i=1}^{Q} \boldsymbol{f}_{i}\right]_{k}=\left[\sum_{i=1}^{Q} \boldsymbol{g}_{i}\right]_{k}=p_{k}$ for $k \in T$,

$$
s_{k}=\alpha p_{k}+(1-\alpha) p_{k}=p_{k} \text { for } k \in T \text {. }
$$

Therefore, $C_{T}$ is convex. Q.E.D.

\section{Proof of projection}

To find the minimum of $\frac{1}{2} \sum_{i=1}^{Q}\left\|\left(\boldsymbol{z}_{\boldsymbol{i}}-\boldsymbol{d}_{i}\right)\right\|^{2}$ subject to $\mathbf{I}_{T}\left(\sum_{j=1}^{Q} \boldsymbol{z}_{j}\right)=\mathbf{I}_{T} \boldsymbol{p}$, we need to minimize

$$
J=\frac{1}{2} \sum_{i=1}^{Q}\left\|\boldsymbol{z}_{i}-\boldsymbol{d}_{i}\right\|^{2}+\lambda^{T} \mathbf{I}_{T}\left(\sum_{j=1}^{Q} \boldsymbol{z}_{j}-\boldsymbol{p}\right)
$$

where $\lambda$ is a $M \times 1$ vector whose element is $\lambda$. Differentiating $J$ gives

$$
\begin{aligned}
\frac{\partial J}{\partial \boldsymbol{z}_{k}} & =\left(\boldsymbol{z}_{k}-\boldsymbol{d}_{k}\right)+\mathbf{I}_{T} \lambda \\
& =0 .
\end{aligned}
$$

This yields to $z_{k}=\boldsymbol{d}_{k}-\mathbf{I}_{T} \boldsymbol{\lambda}$. Since $\boldsymbol{z} \in C_{D}$,

$$
\begin{aligned}
\mathbf{I}_{T} \sum_{j=1}^{Q} \boldsymbol{z}_{j} & =\mathbf{I}_{T} \sum_{j=1}^{Q} \boldsymbol{d}_{j}-Q \mathbf{I}_{T} \boldsymbol{\lambda} \\
& =\mathbf{I}_{T} \boldsymbol{p} .
\end{aligned}
$$

Solving this for $\mathbf{I}_{T} \boldsymbol{\lambda}$ gives $\mathbf{I}_{T} \boldsymbol{\lambda}=-\frac{1}{Q} \mathbf{I}_{T}\left(\boldsymbol{p}-\sum_{j=1}^{Q} \boldsymbol{d}_{j}\right)$. Thus

$$
\boldsymbol{z}_{k}=\boldsymbol{d}_{k}+\frac{1}{Q} \mathbf{I}_{T}\left(\boldsymbol{p}-\sum_{j=1}^{Q} \boldsymbol{d}_{j}\right) .
$$

This means, for the outside treatment region, the dose vector does not change. Therefore, the projection of $\boldsymbol{d}$ is

$$
P_{T} \boldsymbol{d}=\left(\left[P_{T} \boldsymbol{d}\right]_{1}^{T}\left[P_{T} \boldsymbol{d}\right]_{2}^{T} \cdots\left[P_{T} \boldsymbol{d}\right]_{Q}^{T}\right)^{T}
$$

where

$$
\left[P_{T} \boldsymbol{d}\right]_{k}=\boldsymbol{d}_{k}+\frac{1}{Q} \mathbf{I}_{T}\left(\boldsymbol{p}-\sum_{i=1}^{Q} \boldsymbol{d}_{i}\right) .
$$

Q.E.D. 


\section{Appendix C. Proof of convexity and projection of $C_{O}$}

\section{Proof of convexity}

Let $\boldsymbol{f}, \boldsymbol{g} \in C_{O}$. Then from the definition of the set, $C_{O}$,

$$
0 \leqslant \alpha \sum_{i=1}^{Q} c^{T} f_{i} \leqslant \alpha E_{1}
$$

and

$$
0 \leqslant(1-\alpha) \sum_{i=1}^{Q} \boldsymbol{c}^{T} \boldsymbol{g}_{i} \leqslant(1-\alpha) E_{1}
$$

Thus

$$
0 \leqslant \alpha \sum_{i=1}^{Q} \sum_{j \in S} f_{i j}+(1-\alpha) \sum_{i=1}^{Q} \sum_{j \in S} g_{i j} \leqslant \alpha E_{1}+(1-\alpha) E_{1}
$$

which simplifies to

$$
0 \leqslant \alpha \sum_{i=1}^{Q} \boldsymbol{c} f_{i}+(1-\alpha) \sum_{i=1}^{Q} \boldsymbol{g}_{i} \leqslant E_{1} .
$$

Since convexity of $C_{B}$ is already proved, the set, $C_{O}$ is convex. Q.E.D.

\section{Proof of projection}

The projection operator for $C_{O}$ is obtained by minimizing $\frac{1}{2} \sum_{i=1}^{Q}\left\|\boldsymbol{z}_{i}-\boldsymbol{d}_{i}\right\|^{2}$ subject to $\sum_{i=1}^{Q} \boldsymbol{r}^{T} \boldsymbol{z}_{i}=E_{1}$. The problem becomes finding the minimum of the following:

$$
\begin{aligned}
J & =\frac{1}{2} \sum_{i=1}^{Q}\left\|\boldsymbol{z}_{i}-\boldsymbol{d}_{i}\right\|^{2}+\lambda\left(\sum_{i=1}^{Q} \boldsymbol{r}^{T} \boldsymbol{z}_{i}-E_{1}\right) \\
& =\frac{1}{2} \sum_{i=1}^{Q}\left\|\mathbf{A}_{i} \boldsymbol{b}_{i}-\boldsymbol{d}_{i}\right\|^{2}+\lambda\left(\sum_{i=1}^{Q} \boldsymbol{r}^{T} \mathbf{A}_{i} \boldsymbol{b}_{i}-E_{1}\right) .
\end{aligned}
$$

Differentiating it w.r.t. $\boldsymbol{b}_{k}$ gives

$$
\begin{aligned}
\frac{\partial J}{\partial \boldsymbol{b}_{k}} & =\mathbf{A}_{k}^{T}\left(\mathbf{A}_{k} \boldsymbol{b}_{k}-\boldsymbol{d}_{k}\right)+\lambda \mathbf{A}_{k}^{T} \boldsymbol{r} \\
& =0 .
\end{aligned}
$$

Thus $\boldsymbol{b}_{k}=\left(\mathbf{A}_{k}^{T} \mathbf{A}_{k}\right)^{-1}\left(\mathbf{A}_{k}^{T} \boldsymbol{d}_{k}-\lambda \mathbf{A}_{k}^{T} \boldsymbol{r}\right)$. The corresponding dose vector is

$$
\begin{aligned}
\boldsymbol{z}_{k} & =\mathbf{A}_{k} \boldsymbol{b}_{k} \\
& =\mathbf{A}_{k}\left(\mathbf{A}_{k}^{T} \mathbf{A}_{k}\right)^{-1}\left(\mathbf{A}_{k}^{T} \boldsymbol{d}_{k}-\lambda \mathbf{A}_{i}^{T} \boldsymbol{r}\right) \\
& =\mathbf{T}_{k} \boldsymbol{d}_{k}-\lambda \mathbf{T}_{k} \boldsymbol{r}
\end{aligned}
$$

where $\mathbf{T}_{k}=\mathbf{A}_{k}\left(\mathbf{A}_{k}^{T} \mathbf{A}_{k}\right)^{-1} \mathbf{A}_{k}^{T}$. Since $z \in C_{B}$,

$$
\begin{aligned}
\sum_{i=1}^{Q} \boldsymbol{r}^{T} \boldsymbol{z}_{i} & =\sum_{i=1}^{Q}\left(\boldsymbol{r}^{T} \mathbf{T}_{i} \boldsymbol{d}_{i}-\lambda \boldsymbol{r}^{T} \mathbf{T}_{i} \boldsymbol{r}\right) \\
& =E_{1} .
\end{aligned}
$$


Solving for $\lambda$ gives

$$
\lambda=\frac{\sum_{i=1}^{Q} \boldsymbol{r}^{T} \mathbf{T}_{i} \boldsymbol{d}_{i}-E_{1}}{\sum_{i=1}^{Q} \boldsymbol{r}^{T} \mathbf{T}_{i} \boldsymbol{r}} .
$$

Thus

$$
\boldsymbol{z}_{k}=\mathbf{T}_{k} \boldsymbol{d}_{k}-\frac{\sum_{i=1}^{Q} \boldsymbol{r}^{T} \mathbf{T}_{i} \boldsymbol{d}_{i}-E_{1}}{\sum_{i=1}^{Q} \boldsymbol{r}^{T} \mathbf{T}_{i} \boldsymbol{r}} \mathbf{T}_{k} \boldsymbol{r}
$$

Therefore, the projection of $\boldsymbol{d}$ onto $C_{B}$ becomes

$$
P_{O} \boldsymbol{d}=\left(\left[P_{O} \boldsymbol{d}\right]_{1}^{T}\left[P_{O} \boldsymbol{d}\right]_{2}^{T} \ldots\left[P_{O} \boldsymbol{d}\right]_{Q}^{T}\right)^{T}
$$

where

$$
\left[P_{O} \boldsymbol{d}\right]_{k}= \begin{cases}\mathbf{T}_{k}\left(\boldsymbol{d}_{k}+\frac{E_{1}-\sum_{j=1}^{Q} \boldsymbol{r}^{T} \mathbf{T}_{j} \boldsymbol{d}_{j}}{\sum_{j=1}^{Q} \boldsymbol{r}^{T} \mathbf{T}_{j} \boldsymbol{r}} \boldsymbol{r}\right) & \sum_{j=1}^{Q} \boldsymbol{r}^{T} \mathbf{T}_{j} \boldsymbol{d}_{j}>E_{1} \\ \mathbf{T}_{k}\left(\boldsymbol{d}_{k}-\frac{\sum_{j=1}^{Q} \boldsymbol{r}^{T} \mathbf{T}_{j} \boldsymbol{d}_{j}}{\sum_{j=1}^{Q} \boldsymbol{r}^{T} \mathbf{T}_{j} \boldsymbol{r}} \boldsymbol{r}\right) & \sum_{j=1}^{Q} \boldsymbol{r}^{T} \mathbf{T}_{j} \boldsymbol{d}_{j}<0 \\ \mathbf{T}_{k} \boldsymbol{d}_{k} & \text { otherwise. }\end{cases}
$$

Q.E.D.

\section{Appendix D. Proof of convexity and projection of non-negativity beam constraint}

For a specified $\boldsymbol{a}_{k n}$, the convexity of the set $C_{k n}$ in equation (18) follows immediately from the convexity of the set of non-negative numbers.

Consider, then, the projection onto $C_{k n}$. Let $\mathcal{A}_{k n}$ be the column space of $\mathbf{A}_{k n}$ and $\mathcal{A}_{k}$ the column space of $\mathbf{A}_{k}$. As is illustrated in figure A1, the subspace $\mathcal{A}_{k n}$ divides the column space $\mathcal{A}_{k}$ into two halves through the origin. The side on which $\boldsymbol{a}_{k n}$ lies is that half corresponding to positive $b_{k n}$ 's. This half of the space, including the boundary, is the set $C_{k n}$. If a vector does not lie in this half space, the projection onto the half space is equivalent to projection onto $\mathcal{A}_{k n}$ which, of course, is accomplished through the projection matrix $\mathbf{T}_{k n}$. The composite projection in equation (19) results.

Computationally, the projection matrix in equation (20) can be written

$$
\mathbf{T}_{k n}=\mathbf{T}_{k}-\boldsymbol{u}_{k n} \boldsymbol{u}_{k n}^{T}
$$

where the unit vector is

$$
\boldsymbol{u}_{k n}=\frac{\left(\mathbf{I}-\mathbf{T}_{k n}\right) \boldsymbol{a}_{k n}}{\left\|\left(\mathbf{I}-\mathbf{T}_{k n}\right) \boldsymbol{a}_{k n}\right\|} .
$$

The identity follows immediately from the Gram-Schmidt orthogonalization procedure.

\section{References}

Bortfeld T, Burkelbach J, Boesecks R and Schlegal W 1990 Methods of image reconstruction from projections applied to conformation radiotherapy Phys. Med. Biol. 35 1423-34

Brahme A 1988 Optimization of stationary and moving beam radiation therapy techniques Radiother. Oncol. 12 129-40

Bregman L M 1965 Finding the common point of convex sets by the method of successive projections Doki. Akad. Nauk. USSR 162 487-90 
Chen Z, Wang X, Bortfeld T, Mohan R and Reinstein L 1995 The influence of scatter on the design of optimized intensity modulations Med. Phys. 22 1727-33

Cho P S, Lee S, Marks R J II, Oh S, Sutlief S G and Phillips M H Optimization of intensity modulated beams with volume constraints using two methods: cost function minimization and projections onto convex sets Med. Phys. (submitted)

Gubin L G, Polyak B T and Raik E V 1967 The method of projections for finding the common point if convex sets USSR Comput. Math. Phys. 7 1-24

Holmes T W and Mackie T R 1994 A comparison of three inverse treatment planning algorithms Phys. Med. Biol. 39 91-106

Holmes T W, Mackie T R, Simpkin D and Reckwerdt P 1991 A unified approach to the optimization of brachytherapy and external beam dosimetry Int. J. Radiation Oncology Biol. Phys. 20 859-73

Mageras G S and Mohan R 1993 Application of fast simulated annealing to optimization of conformal radiation treatments Med. Phys. 20 639-47

Marks R J II 1997 Alternating projection onto convex sets Deconvolution of Images and Spectra ed P Jansson (San Diego, CA: Academic) pp 476-501

Morrill S M, Lane R G and Rosen I I 1990 Constrained simulated annealing for optimized radiation therapy treatment planning Comput. Methods Prog. Biomed 33 135-44

Rosen I I, Lam K S, Lane R G, Langer M and Morrill S M 1995 Comparison of simulated annealing algorithms for conformal therapy treatment planning Int. J. Radiat. Oncol. Biol. Phys. 33 1091-9

Stark H (ed) 1987 Image Recovery: Theory and Application (Orlando, FL: Academic)

Webb S 1989 Optimization of conformal radiotheraphy dose distributions by simulated annealing Phys. Med. Biol. 34 1349-70

Youla D C and Velasco V 1986 Extensions of a result on the synthesis of signals in the presence of inconsistent constraints IEEE Trans. Circuits Systems 33 465-8

Youla D C and Webb H 1982 Image restoration by method of convex set projections: Part I-Theory IEEE Trans. Med. Imaging 1 81-94 